\title{
BMJ Open Relative risk values of age, acrolein, IL-6 and CRP as markers of periventricular hyperintensities: a cross-sectional study
}

\author{
Arata Abe, Yasuhiro Nishiyama, Mina Harada-Abe, Seiji Okubo, Masayuki Ueda, \\ Masahiro Mishina, Yasuo Katayama
}

To cite: Abe A, Nishiyama Y, Harada-Abe M, et al. Relative risk values of age, acrolein, IL-6 and CRP as markers of periventricular

hyperintensities:

a cross-sectional study. BMJ Open 2014;4: $\mathrm{e} 005598$.

doi:10.1136/bmjopen-2014005598

- Prepublication history for this paper is available online. To view these files please visit the journal online (http://dx.doi.org/10.1136/ bmjopen-2014-005598).

Received 9 May 2014

Revised 4 July 2014

Accepted 24 July 2014

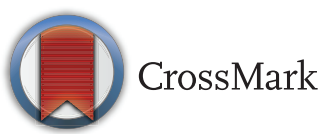

Department of Neurological Science, Graduate School of Medicine, Nippon Medical School, Tokyo, Japan

Correspondence to Dr Arata Abe; abe@nms.ac.jp

\section{ABSTRACT}

Objective: Brain white matter hyperintensities can be divided into periventricular hyperintensity (PVH) and deep-and-subcortical white matter hyperintensity (DSWMH); the former contributes more to cognitive dysfunction and infarction risk. We conducted the present investigation to define the relationship between $\mathrm{PVH}$ and DSWMH.

Design: Cross-sectional study.

Setting: University hospital.

Participants: We prospectively enrolled 228 healthy Japanese volunteers with relative risk values (RRVs) $>0.5$.

Primary outcome measures: We investigated whether it is possible to use the RRV to predict PVH and DSWMH.

Results: Among 228 volunteers, 103 (45.1\%) and $157(68.8 \%)$ exhibited PVH and DSWMH, respectively. Age, body mass index and PVH were significant independent determinants of RRV. A significant OR for $\mathrm{PVH}$ was noted in the highest RRV tertile compared with the lowest, after adjusting for potential confounding factors. A significant $\mathrm{OR}$ for high predicted PVH risk was found for RRV levels as well. Conclusions: Elevated RRV levels were significantly associated with increased predicted PVH, suggesting that measuring the plasma protein-conjugated acrolein, interleukin 6 and $C$ reactive protein levels may be useful for identifying Japanese at high risk for PVH.

\section{INTRODUCTION}

A number of large-scale clinical studies have demonstrated that white matter hyperintensities (WMHs) are associated with high stroke risk. ${ }^{1-3}$ The results of the large-scale, multicentre open trial PICA study ${ }^{4}$ conducted in Japan suggest that the Fazekas-classified periventricular hyperintensities (PVHs) and deep-and-subcortical white matter hyperintensities (DSWMHs) ${ }^{5}$ are related to the risk of symptomatic brain infarction. In the

\section{Strengths and limitations of this study}

Using the relative risk value (RRV) at the clinical level, we investigated to evaluate white matter hyperintensities.

- We provided the first evidence that RRV is associated with periventricular hyperintensity rather than deep-and-subcortical white matter hyperintensity.

- These data are obtained from cases of cautious healthcare in Asian people and may not be applicable to populations of poor health.

Rotterdam Scan study on elderly patients with no history of stroke, conducted by MRI for 4.2 years, the proportional HR of stroke occurrence after adjustment of comorbid factors was 4.7 (95\% CI 2.0 to 11.2) in PVH and 3.6 (CI 1.4 to 9.2 ) in DSWMH. ${ }^{6}$ Unlike DSWMH, PVH is associated with cognitive dysfunction. ${ }^{7}$ In other studies, associations were separately assessed for PVH and DSWMH and was significant only for PVH, which was related to decreased processing speed and executive function. ${ }^{8}{ }^{9}$ Additionally, PVH predicted poorer functional outcome after stroke both in the acute and chronic phases, independently of DSWMH. ${ }^{10}{ }^{11}$ A Chiba University group reported that the relative risk value (RRV) measured based on protein-conjugated acrolein (PCAcro) together with interleukin 6 (IL-6) and C reactive protein (CRP) can be used to predict the stroke risk factors of silent brain infarction, carotid atherosclerosis, and WMH with high sensitivity and specificity. ${ }^{12}$ We measured plasma PCAcro, IL-6 and CRP, and analysed the measurements in conjunction with age to determine whether it is possible to use the RRV to predict PVH and DSWMH. 


\section{MATERIALS AND METHODS}

\section{Subjects and blood sampling}

We examined 228 adult volunteers (78 women and 150 men, aged $65.0 \pm 7.0$ years, range $31-83$ years). All these volunteers were healthy, living independently at home without apparent history of stroke, cardiovascular disease or malignancy. Volunteers with RRV $>0.5$ were enrolled prospectively. Informed consent was provided by each participant, and our study protocol was approved by the Ethics Committees of Nippon Medical School Hospital. Experiments were carried out in accordance with the Declaration of Helsinki principles. Blood samples were collected into tubes containing $3 \mathrm{U} / \mathrm{mL}$ heparin and centrifuged at $1500 \mathrm{~g}$ for $10 \mathrm{~min}$ at $4^{\circ} \mathrm{C}$.

\section{PCAcro, IL-6 and CRP measurements}

Blood samples were drawn from the antecubital vein after overnight fasting. PCAcro ( $N$-(3-formyl-3,4-dehydropiperidino)-lysine (FDPlysine) in protein) was determined as previously described $^{13}$ using an ACR-LYSINE ADDUCT ELISA SYSTEM (NOF Corporation, Tokyo, Japan) and $0.01 \mathrm{~mL}$ plasma. IL-6 and CRP were quantified using an Endogen Human IL-6 ELISA kit (Pierce Biotechnology, Inc, Rockford, Illinois, USA) and a human CRP ELISA kit (Alpha Diagnostic International, San Antonio, Texas, USA), respectively, according to the manufacturers' protocols. After the reaction was terminated, absorbance was measured at $450 \mathrm{~nm}$ using a microplate reader (MTP-800APC, Hitachi, Tokyo, Japan). The biochemical markers from each participant were measured by an investigator who was blinded to the MRI results (Amine Pharma Research Institute, Chiba, Japan). RRV was calculated with artificial neural networks by back propagation method using NEUROSIM/L software V.4 (Fujitsu, Tokyo, Japan). ${ }^{14}$ Using the report by a Chiba University group, we worked out predictive RRV in the range of $0-1,{ }^{12} 15$ with nil as the lowest value, as an index of the degree of tissue damage. Values $>0.5$ were considered to indicate WMH risk.

Standard enzymatic methods were used to measure the levels of serum total cholesterol, triglycerides, creatinine and plasma glucose. Serum high-density lipoprotein (HDL) cholesterol level was measured with a direct method, and serum low-density lipoprotein cholesterol level was calculated using Friedewald's formula in the 228 participants with serum triglyceride levels $<400 \mathrm{mg} / \mathrm{dL}{ }^{16}$ Diabetes was defined as a fasting plasma glucose level $\geq 126 \mathrm{mg} / \mathrm{dL}$ or the use of glucose-lowering medications. Dyslipidaemia was defined as total cholesterol level $\geq 220 \mathrm{mg} / \mathrm{dL}$, HDL cholesterol level $<40 \mathrm{mg} / \mathrm{dL}$ and a triglyceride level $\geq 150 \mathrm{mg} / \mathrm{dL}$, as well as the use of lipid-lowering medications. The estimated glomerular filtration rate (eGFR) was calculated for Japanese men as recommended by the Japanese Society of Nephrology ${ }^{17}$ and represented as: eGFR $\left(\mathrm{mL} / \mathrm{min} / 1.73 \mathrm{~m}^{2}\right)$ $=193 \times$ serum creatinine $e^{-1.094} \times$ age $^{-0.287}$.
Imaging

All 228 participants underwent T1-weighted and T2-weighted MRI and fluid-attenuated inversion recovery (FLAIR) at the Nippon Medical School Hospital, Japan, within 1 month after blood sampling. MRI was performed as described previously. ${ }^{10} \mathrm{PVH}$ and DSWMH were defined as hyperintense areas on T2 and FLAIR images without any abnormality on $\mathrm{T}^{18}$ in patients without neurological signs and/or symptoms. The 228 volunteers were classified into 103 volunteers with $\mathrm{PVH}$ (38 women and 65 men, aged $68.2 \pm 6.0$ years, RRV 0.75 $\pm 0.11)$ and 157 volunteers with WMH (61 women and 96 men, aged $66.7 \pm 5.8$ years, RRV $0.71 \pm 0.12$ ). In more detail, 76 among all the participants had both $\mathrm{PVH}$ and DSWMH, with the other 23 having silent brain infarction. Also, 22 participants had a complication of $\mathrm{PVH}$ and silent brain infarction, while in 20 participants there was complication of DSWMH and silent brain infarction.

\section{Statistics}

All statistical tests were performed using the JMP9.02 software program (SAS Institute, Cary, North Carolina, USA). Continuous variables except for triglyceride levels were expressed as mean $\pm \mathrm{SD}$. Triglyceride levels were transformed to the common logarithm for statistical analysis and are expressed as the geometric mean because of their skewed distribution. Categorical data are expressed as the number of participants (per cent of total). The clinical characteristics for each RRV tertile were compared by analysis of variance (ANOVA) for continuous variables and $\chi^{2}$ test for categorical variables. The RRVs between the two groups were compared by Student $t$ tests or by ANOVA followed by multiple comparisons with the Bonferroni correction between the two groups. Correlations between RRV and other variables were evaluated with Pearson's moment correlation coefficient. Factors with a $\mathrm{p}$ value $<0.05$ as determined by Pearson's correlation analysis were included in a multiple linear regression analysis to identify independent determinants of the RRV. Logistic regression analysis was performed to obtain the ORs for PVH and DSWMH in the three tertiles. All statistical tests were two-sided, and a $\mathrm{p}$ value $<0.05$ was considered as significant.

\section{RESULTS}

The study participants were divided into tertiles according to RRV (0.50-0.62, 0.63-0.79 and 0.80-0.90 from the lowest to highest tertile, respectively). The participants' clinical characteristics are summarised in table 1 . The mean RRV of the entire subject population was 0.71 \pm 0.13 and the mean age was $65 \pm 7$ years. Age, body mass index (BMI), diastolic blood pressure (BP), HDL cholesterol level, triglyceride level, eGFR and current smoking status were significantly different among the groups.

RRVs were significantly higher in participants with older age, lower eGFR or PVH (table 1). A simple correlation analysis showed that RRV was significantly 
Table 1 Characteristics of the study participants due to their stroke risk marker level tertile

\begin{tabular}{|c|c|c|c|c|c|}
\hline \multirow[b]{2}{*}{ Item } & \multirow[b]{2}{*}{ All } & \multicolumn{3}{|c|}{ Silent brain infarction RRV tertile } & \multirow[b]{2}{*}{ p Value* } \\
\hline & & Tertile 1 & Tertile 2 & Tertile 3 & \\
\hline Participants (n) & 228 & 76 & 73 & 79 & - \\
\hline Silent brain infarction relative risk value & $0.71 \pm 0.13$ & $0.51 \pm 0.07 \dagger$ & $0.69 \pm 0.05 \dagger$ & $0.86 \pm 0.03 \dagger$ & १ \\
\hline Age (years) & $65 \pm 7$ & $59 \pm 7$ & $66 \pm 3$ & $70 \pm 6$ & $<0.0001$ \\
\hline Male sex & $150(65.7)$ & $51(67.1)$ & $52(71.2)$ & $47(59.4)$ & 0.299 \\
\hline Body mass index, $\mathrm{kg} / \mathrm{m}^{2}$ & $24.2 \pm 6.4$ & $23.2 \pm 2.9$ & $24.6 \pm 6.3$ & $24.8 \pm 8.6$ & 0.133 \\
\hline Systolic BP, mm Hg & $123 \pm 15$ & $121 \pm 14$ & $122 \pm 14$ & $126 \pm 17$ & 0.275 \\
\hline Diastolic BP, mm Hg & $75 \pm 11$ & $75 \pm 9$ & $75 \pm 10$ & $75 \pm 13$ & 0.947 \\
\hline Hypertension, n (\%) & $81(35.6)$ & $23(30.6)$ & $20(27.4)$ & $38(48.1)$ & 0.015 \\
\hline Total cholesterol, mg/dL & $208 \pm 32$ & $208 \pm 30$ & $208 \pm 32$ & $207 \pm 34$ & 0.903 \\
\hline LDL cholesterol $\ddagger$ mg/dL & $123 \pm 29$ & $123 \pm 25$ & $123 \pm 29$ & $122 \pm 33$ & 0.848 \\
\hline HDL cholesterol, mg/dL & $58 \pm 15$ & $59 \pm 17$ & $57 \pm 13$ & $60 \pm 16$ & 0.385 \\
\hline Triglycerides§, mg/dL & $125(113,138)$ & $141(111,172)$ & $123(105,142)$ & $112(99,124)$ & 0.425 \\
\hline Dyslipidaemia, n (\%) & $55(24.1)$ & $19(25.0)$ & $13(17.8)$ & $23(29.1)$ & 0.259 \\
\hline Fasting plasma glucose, $\mathrm{mg} / \mathrm{dL}$ & $100 \pm 16$ & $100 \pm 14$ & $99 \pm 18$ & $100 \pm 15$ & 0.754 \\
\hline Diabetes, n (\%) & $18(7.8)$ & $3(3.9)$ & $6(8.2)$ & $9(11.3)$ & 0.226 \\
\hline eGFR $\left(\mathrm{mL} / \mathrm{min} / 1.73 \mathrm{~m}^{2}\right)$ & $67.6 \pm 12.5$ & $70.2 \pm 15.3$ & $67.3 \pm 10.6$ & $65.4 \pm 10.6$ & 0.027 \\
\hline Current smoking status, n (\%) & $40(17.5)$ & $16(21.0)$ & $12(16.4)$ & $12(15.1)$ & 0.603 \\
\hline $\mathrm{PVH}, \mathrm{n}(\%)$ & $103(45.1)$ & $18(23.6)$ & $36(49.3)$ & 49 (62.0) & $<0.0001$ \\
\hline DSWMH, n (\%) & $157(68.8)$ & $52(68.4)$ & $50(68.4)$ & $55(69.6)$ & 0.983 \\
\hline \multicolumn{6}{|c|}{$\begin{array}{l}{ }^{*} \text { Analysis of variance or } \chi^{2} \text { test among the ADMA tertile. } \\
\dagger \text { tRange of a minimum-to-maximum stroke risk marker in each tertile. } \\
\text { †n=228. } \\
\text { \$Geometric mean ( } 95 \% \text { Cls). } \\
\text { "IStatistical analysis was not conducted because of the extremely small number o }\end{array}$} \\
\hline
\end{tabular}

correlated with age, systolic BP, eGFR and PVH (table 2). Multiple linear regression analysis indicated that BMI $(\beta=0.0026, p=0.044)$ and PVH $(\beta=0.0380, p<0.0001)$ were significant independent determinants of RRV.

The results of logistic regression analysis of the association between PVH and RRV are shown in table 3 . Significant, unadjusted ORs for PVH were noted in the third RRV tertile $(5.26(95 \%$ CI 2.66 to 10.78$)$, $\mathrm{p}<0.0001)$, compared with the first tertile. After adjusting for model 1 (BMI, systolic BP, triglycerides, eGFR and current smoking status), the ORs in the third RRV tertile remained significant $(4.75 \quad(95 \%$ CI 2.33 to $10.05), \mathrm{p}<0.0001)$. After adjusting for model 2 , we found that the OR in the third RRV tertile 3 was significant (5.26 (95\% CI 2.65 to 10.83$), \mathrm{p}<0.0001)$. A significant relationship was observed between RRV and PVH $(\mathrm{p}<0.05)$, but no such significance was found between RRV and DSWMH (figure 1).

\section{DISCUSSION}

The present study demonstrated a significant, positive correlation between RRV and PVH in healthy Japanese volunteers. Notably, the highest RRV level tertile showed a significantly higher OR for a high predicted PVH risk in comparison to the lowest tertile, even after adjusting for multiple confounding factors. These results suggest that RRV is associated with the estimated risk of PVH in healthy Japanese volunteers. A number of clinical and epidemiological studies have examined the predictive value of RRV for the presence of WMH. ${ }^{12}{ }^{15}$ However, those studies assessed WMH prevalence; no studies have shown any significant association of RRV with PVH and DSWMH separately. In this regard, our results raise the possibility that RRV predicts the risk of $\mathrm{PVH}$ in the healthy Japanese population. With respect to age, these biochemical markers provide a good index of the presence of tissue damage related to PVH.

Recent studies focused on WMH location have reported that functional impairment within 1-3 months after stroke correlated with PVH but not with DSWMH. ${ }^{10} 19$ PVH WMH, especially PVH, has impacts on early functional recovery after ischaemic stroke regardless of the initial stroke severity and other cardiovascular risk factors. ${ }^{11}$ Other groups found a significant association between PVH and decreases in processing speed and executive function, but there was no such relationship with DSWMH. ${ }^{8}{ }^{9}$ Why PVH and DSWMH have different relationships with stroke outcome remains unclear, but several theories have been put forward. DSWMH predominantly disrupts short association fibres that link adjacent gyri, while PVH affects long association fibres that connect the more distant cortical areas. $^{20}$ Thus, lesions in various white matter locations may disconnect from different neural networks that affect neural repair processes after stroke. ${ }^{21}$ In addition, 
Table 2 Correlation coefficients and multiple linear regression analysis of relative risk value with the clinical parameters

\begin{tabular}{|c|c|c|c|c|}
\hline \multirow[b]{2}{*}{ Item } & \multicolumn{2}{|l|}{ Simple correlation analysis } & \multicolumn{2}{|c|}{ Multiple linear regression analysis } \\
\hline & Correlation coefficient $(r)$ & p-Value & $\begin{array}{l}\text { Standardised regression } \\
\text { coefficient }(\beta)\end{array}$ & p Value \\
\hline Age & 0.60 & $<0.0001$ & * & \\
\hline Male sex & 0.05 & 0.347 & $\dagger$ & \\
\hline BMI & 0.11 & 0.075 & $\dagger$ & \\
\hline Systolic BP & 0.14 & 0.026 & 0.0009 & 0.078 \\
\hline Diastolic BP & 0.05 & 0.382 & $\dagger$ & \\
\hline Total cholesterol & -0.03 & 0.618 & $\dagger$ & \\
\hline LDL cholesterol $\ddagger$ & -0.02 & 0.669 & $\dagger$ & \\
\hline HDL cholesterol & 0.02 & 0.731 & $\dagger$ & \\
\hline Triglycerides $\S$ & -0.10 & 0.130 & $\dagger$ & \\
\hline Fasting plasma glucose & 0.01 & 0.841 & $\dagger$ & \\
\hline eGFR & -0.13 & 0.041 & -0.0009 & 0.173 \\
\hline Current smoking status (Yes=1) & -0.10 & 0.115 & $\dagger$ & \\
\hline $\mathrm{PVH}(\mathrm{Yes}=1)$ & 0.26 & $<0.0001$ & 0.0384 & $<0.0001$ \\
\hline DSWMH (Yes=1) & 0.02 & 0.689 & $\dagger$ & \\
\hline
\end{tabular}

PVHs are related to diminished cerebral vasomotor reactivity and subsequent occurrence of cerebral hypoperfusion, ${ }^{22}$ while DSWMHs are generally associated with microangiopathy. ${ }^{23}$ It is clear that regional hypoperfusion is a good predictor of functional outcome. ${ }^{24}$ These findings shed light on why PVH can predict functional stroke outcome and specific cognitive functions. ${ }^{11}$

Acrolein induces IL-6 production in astrocytes, macrophages and endothelial cells, while IL-6 induces CRP production in hepatocytes. Then, CRP stimulates IL-6 production and IL-6 decreases acrolein toxicity. ${ }^{25}$ Acrolein was thought to be one of the toxic compounds produced from unsaturated fatty acids by active oxygen species such as superoxide anion radicals, hydrogen peroxide and hydroxyl radicals. ${ }^{13}$ These findings may partially explain the pathophysiological mechanisms underlying the association between $\mathrm{PVH}$ and the three biomarkers assessed in the present study. Further investigation will be needed for a better understanding of their inter-relationship.

Our multiple linear regression analysis showed that RRV was independently associated with BMI and PVH. Although obesity is believed to be an independent cardiovascular risk factor, ${ }^{26}$ it is still controversial whether
BMI is a significant risk factor for stroke. ${ }^{27}$ BMI was previously reported to be correlated with high RRV, ${ }^{29}$ which may be caused by vascular degeneration and endothelial dysfunction associated with hypertension and metabolic disorders. Patients with metabolic syndrome are generally defined as those who have abdominal obesity and two additional metabolic disorders including hypertension, dyslipidaemia and hyperglycaemia. 3031

Our study has some potential limitations. Because it was a cross-sectional investigation, we could not determine a causal relationship between increased RRV and PVH risk. In addition, the population included healthy Japanese volunteers only. Therefore, it is unclear whether the results can be extrapolated to other populations of poor health, patients with cardiovascular diseases or other ethnic groups. Despite these potential limitations, our findings support the conclusion that elevated RRV is significantly associated with PVH in healthy Japanese volunteers. These results suggest that RRV measurement may be useful for identifying $\mathrm{PVH}$ in the general population. This would allow clinicians to follow patients who may be at risk for stroke and cognitive dysfunction.

Table 3 Unadjusted and adjusted PVH ORs in each silent brain infarction relative risk value tertile

\begin{tabular}{|c|c|c|c|c|c|c|}
\hline \multirow[b]{2}{*}{ Item } & \multicolumn{3}{|c|}{ Unadjusted } & \multicolumn{3}{|c|}{ Adjusted* } \\
\hline & $\overline{\text { OR }}$ & $95 \% \mathrm{Cl}$ & p Value & OR & $95 \% \mathrm{Cl}$ & p Value \\
\hline Tertile 1 & 1.00 & Reference & - & 1.00 & Reference & - \\
\hline Tertile 2 & 3.13 & 1.57 to 6.41 & 0.0014 & 3.01 & 1.50 to 6.20 & 0.0018 \\
\hline Tertile 3 & 5.26 & 2.66 to 10.78 & $<0.0001$ & 4.87 & 2.43 to 10.08 & $<0.0001$ \\
\hline
\end{tabular}


A

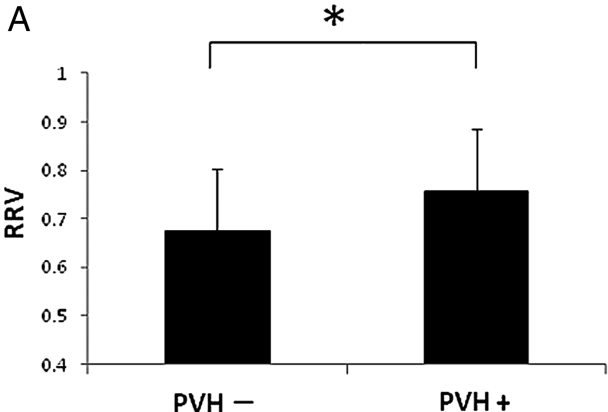

B

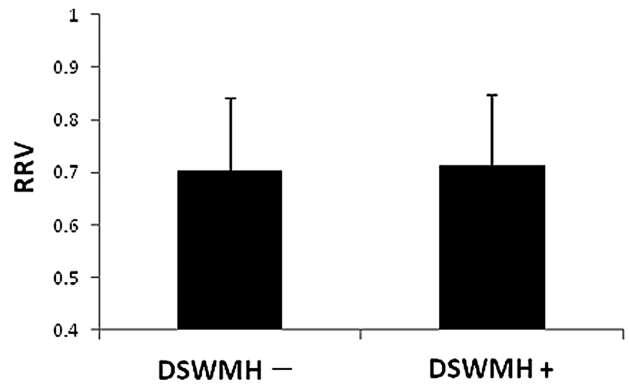

Figure 1 (A) Correlation between the relative risk value (RRV) and the periventricular hyperintensity (PVH). (B) Correlation between the RRV and DSWMH. *Significant at $p<0.05$.

Acknowledgements The authors thank Dr Mari Adachi and Dr Sadaji Kura at Katsushika Health Center for assisting with the data analyses.

Contributors AA was involved in conception and design of the study. YN, $\mathrm{MH}-\mathrm{A}, \mathrm{SO}$ and $\mathrm{MU}$ participated in analysis and interpretation. AA wrote the article. YN, MM and YK critically revised the article. YN, MH-A, SO, MU, MM and $Y K$ were involved in final approval of the article. Statistical analysis was carried out by YN and MM. AA was overall responsible for the study.

Funding This research received no specific grant from any funding agency in the public, commercial or not-for-profit sectors.

Competing interests None

Patient consent Obtained

Ethics approval Obtained.

Provenance and peer review Not commissioned; externally peer reviewed.

Data sharing statement No additional data are available.

Open Access This is an Open Access article distributed in accordance with the Creative Commons Attribution Non Commercial (CC BY-NC 4.0) license, which permits others to distribute, remix, adapt, build upon this work noncommercially, and license their derivative works on different terms, provided the original work is properly cited and the use is non-commercial. See: http:// creativecommons.org/licenses/by-nc/4.0/

\section{REFERENCES}

1. Wong TY, Klein R, Sharrett AR, et al. Cerebral white matter lesions, retinopathy, and incident clinical stroke. JAMA 2002;288:67-74.

2. Gouw AA, van der Flier WM, Fazekas $F$, et al. Progression of white matter hyperintensities and incidence of new lacunes over a 3-year period: the Leukoaraiosis and Disability study. Stroke 2008:39:1414-20.

3. Bokura H, Yamaguchi S, Kobayashi S. Electrophysiological correlates for response inhibition in a Go/NoGo task. Clin Neurophysiol 2001;112:2224-32.

4. Shinohara $\mathrm{Y}$, Tohgi $\mathrm{H}$, Hirai $\mathrm{S}$, et al. Effect of the Ca antagonist nilvadipine on stroke occurrence or recurrence and extension of asymptomatic cerebral infarction in hypertensive patients with or without history of stroke (PICA Study). 1. Design and results at enrollment. Cerebrovasc Dis 2007:24:202-9.

5. Schmidt R, Fazekas F, Kapeller P, et al. MRI white matter hyperintensities: three-year follow-up of the Austrian Stroke Prevention Study. Neurology 1999;53:132-9.

6. Vermeer SE, Hollander M, van Dijk EJ, et al. Silent brain infarcts and white matter lesions increase stroke risk in the general population: the Rotterdam Scan Study. Stroke 2003;34:1126-9.

7. Fukuda $\mathrm{H}$, Kobayashi $\mathrm{S}$, Okada $\mathrm{K}$, et al. Frontal white matter lesions and dementia in lacunar infarction. Stroke 1990;21:1143-9.

8. van den Heuvel DM, ten Dam VH, de Craen AJ, et al. Increase in periventricular white matter hyperintensities parallels decline in mental processing speed in a non-demented elderly population. J Neurol Neurosurg Psychiatry 2006;77:149-53.

9. Debette $S$, Bombois S, Bruandet A, et al. Subcortical hyperintensities are associated with cognitive decline in patients with mild cognitive impairment. Stroke 2007;38:2924-30.
10. Kissela B, Lindsell CJ, Kleindorfer D, et al. Clinical prediction of functional outcome after ischemic stroke: the surprising importance of periventricular white matter disease and race. Stroke 2009;40:530-6.

11. Liou LM, Chen CF, Guo YC, et al. Cerebral white matter hyperintensities predict functional stroke outcome. Cerebrovasc Dis 2010;29:22-7.

12. Yoshida M, Tomitori $\mathrm{H}$, Machi $\mathrm{Y}$, et al. Acrolein, IL-6 and CRP as markers of silent brain infarction. Atherosclerosis 2009; 203:557-62.

13. Uchida K, Kanematsu M, Morimitsu Y, et al. Acrolein is a product of lipid peroxidation reaction. Formation of free acrolein and its conjugate with lysine residues in oxidized low density lipoproteins. J Biol Chem 1998;273:16058-66.

14. Ellenius J, Groth T, Lindahl B, et al. Early assessment of patients with suspected acute myocardial infarction by biochemical monitoring and neural network analysis. Clin Chem 1997;43:1919-25.

15. Yoshida M, Higashi K, Kobayashi E, et al. Correlation between images of silent brain infarction, carotid atherosclerosis and white matter hyperintensity, and plasma levels of acrolein, IL-6 and CRP. Atherosclerosis 2010;211:475-9.

16. Friedewald WT, Levy RI, Fredrickson DS. Estimation of the concentration of low-density lipoprotein cholesterol in plasma, without use of the preparative ultracentrifuge. Clin Chem 1972;18:499-502.

17. Matsuo S, Imai E, Horio M, et al. Revised equations for estimated GFR from serum creatinine in Japan. Am J Kidney Dis 2009;53:982-92.

18. Fazekas $\mathrm{F}$, Kleinert $\mathrm{R}$, Offenbacher $\mathrm{H}$, et al. The morphologic correlate of incidental punctate white matter hyperintensities on MR images. AJNR Am J Neuroradiol 1991;12:915-21.

19. Kang HJ, Stewart R, Park MS, et al. White matter hyperintensities and functional outcomes at 2 weeks and 1 year after stroke. Cerebrovasc Dis 2013;35:138-45.

20. Brodal P. The central nervous system: structure and function. 3rd edn. Oxford: Oxford University Press, 2004.

21. Chollet F, DiPiero V, Wise RJ, et al. The functional anatomy of motor recovery after stroke in humans: a study with positron emission tomography. Ann Neurol 1991;29:63-71.

22. Gerdes VE, Kwa VI, ten Cate $\mathrm{H}$, et al. Cerebral white matter lesions predict both ischemic strokes and myocardial infarctions in patients with established atherosclerotic disease. Atherosclerosis 2006;186:166-72.

23. Fazekas $\mathrm{F}$, Kleinert $\mathrm{R}$, Offenbacher $\mathrm{H}$, et al. Pathologic correlates of incidental MRI white matter signal hyperintensities. Neurology 1993:43:1683-9.

24. Giubilei F, Lenzi GL, Di Piero V, et al. Predictive value of brain perfusion single-photon emission computed tomography in acute ischemic stroke. Stroke 1990;21:895-900.

25. Saiki R, Hayashi D, Ikuo Y, et al. Acrolein stimulates the synthesis of IL-6 and C-reactive protein (CRP) in thrombosis model mice and cultured cells. J Neurochem 2013;127:652-9.

26. Poirier P, Giles TD, Bray GA, et al. Obesity and cardiovascular disease: pathophysiology, evaluation, and effect of weight loss: an update of the 1997 American Heart Association Scientific Statement on Obesity and Heart Disease from the Obesity Committee of the Council on Nutrition, Physical Activity, and Metabolism. Circulation 2006; 113:898-918.

27. Haapaniemi H, Hillbom M, Juvela S. Lifestyle-associated risk factors for acute brain infarction among persons of working age. Stroke 1997;28:26-30. 
28. Rexrode $\mathrm{KM}$, Hennekens $\mathrm{CH}$, Willett WC, et al. A prospective study of body mass index, weight change, and risk of stroke in women. JAMA 1997;277:1539-45.

29. Yoshida M, Mizoi M, Saiki R, et al. Relationship between metabolic disorders and relative risk values of brain infarction estimated by protein-conjugated acrolein, IL-6 and CRP together with age. Clin Chim Acta 2011;412:339-42.
30. Ninomiya JK, L'Italien G, Criqui MH, et al. Association of the metabolic syndrome with history of myocardial infarction and stroke in the Third National Health and Nutrition Examination Survey. Circulation 2004;109:42-6.

31. Kwon HM, Kim BJ, Lee SH, et al. Metabolic syndrome as an independent risk factor of silent brain infarction in healthy people. Stroke 2006;37:466-70. 